\title{
THE RESPONSE OF TYPE H SUPERCONDUCTORS TO TRAPEZOIDAL MAGNETIC FIELDS
}

\author{
L. J. M. van de KLUNDERT, E. A. GIJSBERTSE and H. P. van de BRAAK \\ Twente University of Technology, Department of Applied Physics, P.O. Box 217, Enschede, The Netherlands
}

Received 30 August 1977

\begin{abstract}
The inductive response of type II superconductors to applied fields with trapezoidal time dependence is described for samples with a slab geometry. An extension of the critical state model which accounts for the observed waveform of the voltage, induced in the pick-up coil, is given. Typical experiments on Nb-slabs with weak pinning and flux flow in fields with both small and large amplitudes are reported.
\end{abstract}

\section{Introduction}

The knowledge of the behaviour of type II superconductors in time dependent magnetic fields of large amplitudes is of great interest for their application in, for instance, transport cables and machines. The only model used so far for the description of losses in these superconductors is the critical state model (CSM) [1-3]. The main parameter of this quasi-static model is the critical current density $j_{\mathrm{c}}$. The time dependent behaviour, however, may differ considerably from the quasi-static behaviour because over-critical current densities, due to flux flow, will occur inside the superconductor. When these flux flow effects are dominated by the pinning of flux lines, they may be neglected and the CSM gives a good description of the time dependent behaviour also. A more detailed description of the CSM is given in section 2.

The induction method is a powerful technique for determining the parameters used in the CSM. The waveform of the voltage induced in a pick-up coil wound around the sample is determined by:

(a) the magnetic properties of the material, which are included in the CSM;

(b) the geometry of the sample; and

(c) the waveform of the applied field.

Usually the behaviour of superconductors is investigated for cylindrical samples in sinusoidal fields superimposed on a static field. A detailed analysis of the observed waveform and its amplitude dependence $[3,4]$ then may lead to the determination of the flux distribution inside the sample. From this the critical current density $j_{\mathrm{c}}$ and its position dependence can be obtained. In our investigations the response of slabs subject to alternating fields of trapezoidal waveform was studied. In contrast to a sinusoidal field a trapezoidal waveform provides a more sensitive method for observing the presence of flux flow since only discrete values of the rate of change $\mathrm{d} B / \mathrm{d} t$ (either finite or zero) are used. In general the advantage of the use of trapezoidal fields is that various effects, such as surface screening and flux flow, show up at different time intervals during one cycle, whereas they mix up and mask each other in the sinusoidal case. An advantage of using a slab geometry in trapezoidal fields is that, assuming the absence of flux flow, the flux distribution $B(x)$ inside the slab (for the coordinate system used, see fig. 1) can be seen directly from the observed waveform. The method will be discussed in section 3.

Measurements were done on $\mathrm{Nb}$-slabs. Three types of waveforms were applied:

(a) a trapezoidal field of small amplitude $b_{0}$ superposed on a static field $B_{0}$;

(b) a trapezoidal field of large amplitude $\left(b_{0}<0.5 \mathrm{~T}\right)$; and

(c) a sinusoidal field of large amplitude $\left(b_{0}^{\prime}<0.5 \mathrm{~T}\right)$ In this paper we will restrict ourselves to the first case although also some results for large amplitudes are given for comparison. Preliminary results have already been reported [5,6]. A more detailed analysis of the large amplitude experiments will be published separately. 


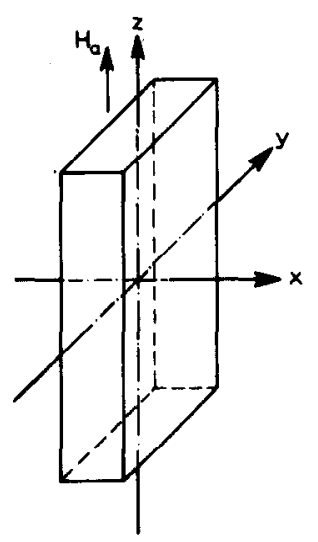

Fig. 1. The coordinate system with sample.

In section 4 the equipment used in the experiments is described. Section 5 gives the experimental results and a discussion on the basis of the theory given in the sections 2 and 3 .

\section{The critical state model (CSM) and the determina- tion of critical current densities}

In the framework of the CSM a local average induction $B$ and a local average field intensity $H$ are defined at every position in the bulk of the sample. It is assumed here that the distance over which $B$ and $H$ vary is large compared with the spacing between neighbouring flux lines. It is also assumed that $B$ and $H$ are connected by the relation $B=\mu H$.

Quasi-static hysteresis effects in type II superconductors may be caused by two different mechanisms:

(a) a surface barrier, accounting for superheating and supercooling, which delays flux entry and flux exit at the surface, and/or

(b) the pinning of flux lines at deformations and impurities in the bulk of the sample as well as at the surface.

We will incorporate both these mechanisms in the CSM. In time dependent fields flux flow and flux creep also will contribute to the hysteresis. These viscous effects will not be included in our model, but their contribution will be treated in the following section. Apart from the mechanisms mentioned above, the actual shape of the hysteresis curve is also influenced by the sample geometry, the roughness of the surface and the distribution of the pinning centres in the sample.

In the case of a sample with a perfectly flat surface and an ideal flux line lattice caused by an external magnetic field parallel to the surface, the flux density just inside the flux line free surface layer is usually assumed to take its equilibrium value $B_{\text {eq }}\left(H_{\mathrm{a}}\right)$ as defined by the reversible magnetization curve (see fig. 2); $H_{\mathrm{a}}$ is the field intensity just outside the sample. The differences in $B$ and in $H$ across the sample surface are caused by the Meissner current which flows in this narrow surface layer. In this case the Meissner current in itself does not give rise to any irreversibilities, but it has been pointed out that superheating and supercooling of the Meissner state and of the mixed state may cause hysteresis. Even between $H_{\mathrm{c}_{2}}$ and $H_{\mathrm{c}_{3}}$ the net surface current may differ from its equilibrium value zero. For the pure Meissner state superheating was calculated by various authors by computing the energy of a single vortex line near the surface, using a method of images $[7,8]$ or solving the one dimensional G.L.-equations $[9,10]$. For the mixed state both superheating and supercooling were discussed by, for instance, Ullmaier [11] and Clem [12]. These effects can be included in the model by replacing $B_{\text {eq }}\left(H_{\mathrm{a}}\right)$ by $B_{\text {en }}\left(H_{\mathrm{a}}\right)$ in the case of superheating and by $B_{\mathrm{ex}}\left(H_{\mathrm{a}}\right)$ for supercooling. For high $\kappa$ superconductors Clem [12], using the method of images, calculated $B_{\mathrm{en}}\left(H_{\mathrm{a}}\right)=\mu_{0}\left(H_{\mathrm{a}}^{2}-H_{\mathrm{s}}^{2}\right)^{\frac{1}{2}}$ and

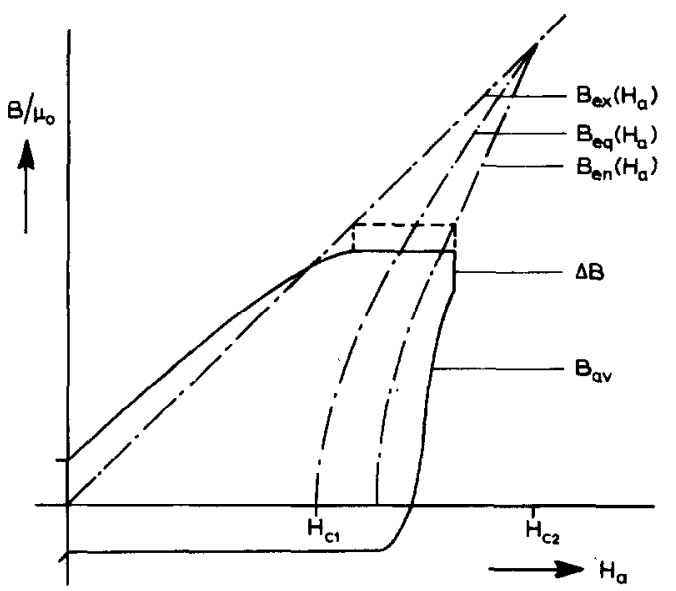

Fig. 2. Schematical $B$ vs. $H_{\mathrm{a}}$ curve showing various parameters of the critical state model (CSM). Also, $\Delta B$ corresponding to the flux flow conductivity $\sigma_{\mathrm{ff}}$ is indicated. 
$B_{\text {ex }}\left(H_{\mathrm{a}}\right)=\mu_{0} H_{\mathrm{a}}$, in which $H_{\mathrm{s}}$ is the superheating field of the Meissner state. The various parameters mentioned above are shown in fig. 2. Between $H_{\mathrm{c}_{2}}$ and $H_{\mathrm{c}_{3}}$ the bulk of the material is already in the normal state. The existence of non-zero Meissner currents was treated phenomenologically by, for instance, Fink [13] and was calculated from the G.L.-equations by Voetmann Christiansen and Smith [14].

In the non-ideal case of a rough surface, the superheating and supercooling effects, as treated above, become less pronounced due to demagnetization. Flux lines will penetrate the surface and one can no longer speak of a flux line free surface layer. Moreover, Akhmedov et al. [15] have pointed out that the superconducting surface layer above $H_{\mathrm{c}_{2}}$ breaks up in a periodic structure of flux lines with a period depending on the angle between the applied field and the surface. This may result in a non-zero net current which cannot be distinguished from the superheating and supercooling effect. It may be noted that a similar effect may arise from an inhomogeneous applied field. In actual cases it is therefore pertinent to describe the delay of the motion of flux lines across the sample surface in the absence of pinning by effective relations $B_{\text {en }}\left(H_{\mathrm{a}}\right)$ and $B_{\text {ex }}\left(H_{\mathrm{a}}\right)$. Quantitative approximations of these effective $B_{\mathrm{en}}$ and $B_{\mathrm{ex}}$ expressions are not available.

The second mechanism causing hysteresis is the pinning of flux lines at deformations and impurities in the bulk of the sample or at the surface (surface pinning). The pinning can be described by a critical current density $j_{\mathrm{c}}$ which is a function of $B$; the variation of $H$ inside the sample is governed by Maxwells equation $\bar{\nabla} \times \bar{H}=\bar{j}_{\mathrm{c}}$. The ideal case of a sample with perfectly flat surfaces and a homogeneous distribution of pinning centres was treated extensively by Bean [1] and London [2]. The critical current density then is constant and parallel to the surface. Inhomogeneity of this distribution in the direction perpendicular to the surface, caused by surface treatment, can be accounted for by allowing $j_{\mathrm{c}}$ to depend on the distance to the surface. Although variations in the permeability $\mu$ with position also may be caused by these deformations, assumptions about a positiondependent $B=\mu H$ relation have never been made. However, in the electrodynamic theory of superconductivity $\mu$ and $j_{\mathrm{c}}$ only occur in the product $\mu j_{\mathrm{c}}$ which is the analogon of the product $\mu \sigma$ for normal metals (see [16]). For this reason a distinction between a position-dependent $\mu$ and a position-dependent $j_{\mathrm{c}}$ cannot be made. Therefore we shall assume $\mu$ to be a constant equal to $\mu_{0}$ so $B=\mu_{0} H$.

From these two concepts, pinning of flux lines and surface screening, the average induction $B_{\mathrm{av}}$ in the sample can be determined, accounting for the dimensions of the sample and its magnetic history (see fig. 2). It is important to remark that for large amplitude experiments the average induction in an increasing field will be smaller than the induction $B_{\text {en }}\left(H_{\mathrm{a}}\right)$ at the surface due to pinning. Analogously, in a decreasing field, $B_{\text {av }}$ will become larger than $B_{\text {ex }}\left(H_{\mathrm{a}}\right)$. As a consequence the $B_{\text {en }}\left(H_{\mathrm{a}}\right)$ and $B_{\text {ex }}\left(H_{\mathrm{a}}\right)$ curves will fall inside the hysteresis loop of $B_{\mathrm{av}}$.

In practical cases additional effects have to be accounted for. First, the flux line lattice may be distorted by the inhomogeneous distribution of pinning sites inside the superconductor and by a rough surface. Secondly the roughness of the surface itself may contribute to the pinning since the motion of flux lines will be obstructed at pinning sites at the surface. The distortion of the flux line lattice can be described by a critical current density which is no longer parallel to the surface and depends on the $y$ and $z$-coordinates as well. In experimental situations only the $x$-dependence of an effective critical current density $j_{\mathrm{c}}$, parallel to the surface can be determined. This effective $j_{\mathrm{c}}$ accounts only for the behaviour of the sample averaged over the volume of the pick-up coil although due to the elastic and viscous properties of the distorted flux line lattice the distribution of pinning sites throughout the whole sample plays a role. It may be noted that the presence of surface pinning sites may also influence the occurrence of metastable states of superheating and supercooling. Therefore the effective $B_{\text {en }}$ and $B_{\text {ex }}$ relations must account not only for surface roughness but for the presence of surface pinning sites as well.

In this way a distinction is made between the delay of the motion of flux lines in varying external fields, described by the $B_{\text {en }}\left(H_{\mathrm{a}}\right)$ and $B_{\text {ex }}\left(H_{\mathrm{a}}\right)$ relations, and the distortion of the flux line lattice, described by the effective critical current density $j_{\mathrm{c}}(x)$.

Several methods for measuring this position dependence have been proposed, all requiring experi- 
ments in small ac fields superimposed on a dc field to ensure that within the applied field range $j_{\mathrm{c}}$ may be considered independent of $B$. It should be noted that these methods are all based on the validity of the CSM, i.e. the absence of flux flow. Using a phasesensitive detector, Campbell [17] obtained flux profiles by measuring the in-phase component of the induced voltage as a function of the amplitude of the applied ac field. Rollins et al. [4] showed that it is possible to calculate the flux distribution from the waveform of the induced voltage. In a previous paper [5] the present authors indicated that it is much more convenient to investigate the response of slabs subject to trapezoidal fields rather than cylinders in a sinusoidal ac field. The latter method will be treated in more detail in the next section.

\section{The trapezoidal waveform}

In this section a quantitative analysis is given of the response of a superconducting slab of thickness $2 d$ in a trapezoidal ac field. This analysis is entirely based on the assumption that the behaviour of the sample obeys the CSM as it was described in the previous section. So it is assumed that only an effective critical current density parallel to the surface exists: $j_{\mathrm{c}}=j_{\mathrm{c}}\{B(x), x\} ; j_{\mathrm{c}}$ accounts for bulk pinning as well as surface pinning. Surface screening effects will be included and described in terms of a $B_{\text {en }}\left(H_{\mathrm{a}}\right)$ and a $B_{\text {ex }}\left(H_{\mathrm{a}}\right)$ relation. Moreover, it will be demonstrated that under certain conditions it is possible to show the flux distribution inside a superconducting slab directly by examining the response of the sample in a small ac field with amplitude $h_{0}$ superimposed on a static field $H_{0}$.

After decreasing the external field from its maximum value $H_{0}+h_{0}$ to its minimum $H_{0}-h_{0}$, the induction just inside the surface layer will be given by $B_{\text {ex }}\left(H_{0}-h_{0}\right)$. When the field is raised again this value and also the complete flux distribution inside the slab will be unchanged until the external field $H_{\mathrm{a}}$ reaches the value $H_{\mathrm{a} 0}$ for which $B_{\mathrm{en}}\left(H_{\mathrm{a} 0}\right)$ $=B_{\text {ex }}\left(H_{0}-h_{0}\right)$. In the corresponding time interval the voltage $U(t)$ induced in the pick-up coil, which is proportional to the $y$-component of the electrical field strength $E(t)$ at the surface, equals zero. (In a more detailed analysis of superheating and super- cooling [12] a variation of the surface current is connected to a variation of the London penetration depth $\lambda_{T}$ [9]. This is related to a change of the total flux in the surface region, so $E(t)$ is not exactly equal to zero. According to London's equation this corresponds to the variation of the supercurrent.) From this interval of the $E$ vs. $H_{\mathrm{a}}$ curve information about the $B_{\text {en }}\left(H_{\mathrm{a}}\right)$ and $B_{\text {ex }}\left(H_{\mathrm{a}}\right)$ curves can be obtained provided the critical current density $j_{\mathrm{c}}$ and its position dependence are known and the applied field is of sufficiently large amplitude. We will return to this point later.

When the external field is increased further, the induction just inside the surface layer is given by $B_{\text {en }}\left(H_{\mathrm{a}}\right)$ exceeding $B_{\text {ex }}\left(H_{0}-h_{0}\right)$. As a consequence flux changes will occur in the region $x_{\mathrm{t}}\left(H_{\mathrm{a}}\right) \leqslant x \leqslant d$; in this region the flux distribution is determined by the external field $H_{\mathrm{a}}$ and the fact that the critical current density has changed sign. For $0 \leqslant x \leqslant x_{t}$ the flux distribution will be unchanged. So we have:

$$
\begin{aligned}
& B^{-}\left(x, H_{\mathrm{a}}\right)=B_{\mathrm{ex}}\left(H_{0}-h_{0}\right) \\
& +\mu_{0} \int_{x}^{d} j_{\mathrm{c}}\left\{B^{-}\left(x^{\prime}, H_{0}-h_{0}\right), x^{\prime}\right\} \mathrm{d} x^{\prime}, \\
& \quad \text { for } 0 \leqslant x \leqslant x_{\mathrm{t}}, \\
& B^{+}\left(x, H_{\mathrm{a}}\right)=B_{\mathrm{en}}\left(H_{\mathrm{a}}\right)-\mu_{0} \int_{x}^{d} j_{\mathrm{c}}\left\{B^{+}\left(x^{\prime}, H_{\mathrm{a}}\right), x^{\prime}\right\} \mathrm{d} x^{\prime}, \\
& \quad \text { for } x_{\mathrm{t}} \leqslant x \leqslant d,
\end{aligned}
$$

where $B^{-}$and $B^{+}$are the flux distributions in decreasing and increasing field respectively; $x_{t}$ is defined by the relation

$B^{+}\left(x_{t}, H_{\mathrm{a}}\right)=B^{-}\left(x_{\mathrm{t}}, H_{\mathrm{a}}\right)=B^{-}\left(x_{t}, H_{0}-h_{0}\right)$.

The parameters used in the description above are illustrated in fig. 3 . The total flux per unit width inside the sample is obtained by the integration

$$
\phi\left(x_{\mathrm{t}}, H_{\mathrm{a}}\right)=2 \int_{0}^{d} B\left(x, H_{\mathrm{a}}\right) \mathrm{d} x
$$




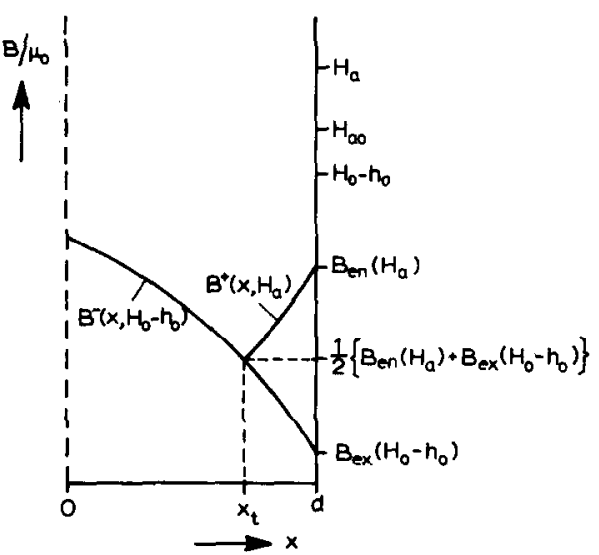

Fig. 3. Flux profiles at two times during the ac cycle: before the increase of the external field starts and for $H_{\mathrm{a}}<H_{\mathrm{a} 0}$ the profile is given by $B^{-}\left(x, H_{0}-h_{0}\right)$. For $H_{\mathrm{a}}>H_{\mathrm{a} 0}$ the profile is given by $B^{-}\left(x, H_{0}-h_{0}\right)$ for $0<x<x_{t}$ and by $B^{+}\left(x, H_{a}\right)$ for $x_{\mathrm{t}}<x<d$.

$$
\begin{aligned}
& =2\left[B_{\mathrm{ex}}\left(H_{0}-h_{0}\right) x_{\mathrm{t}}+B_{\mathrm{en}}\left(H_{\mathrm{a}}\right)\left(d-x_{\mathrm{t}}\right)\right. \\
& +\mu_{0} \int_{0}^{x_{\mathrm{t}}}\left(\int_{x}^{d} j_{\mathrm{c}}\left\{B^{-}\left(x^{\prime}, H_{0}-h_{0}\right), x^{\prime}\right\} \mathrm{d} x^{\prime}\right) \mathrm{d} x \\
& \left.-\mu_{0} \int_{x_{\mathrm{t}}}^{d}\left(\int_{x}^{d} j_{\mathrm{c}}\left\{B^{+}\left(x^{\prime}, H_{\mathrm{a}}\right), x^{\prime}\right\} \mathrm{d} x^{\prime}\right) \mathrm{d} x\right] .
\end{aligned}
$$

The electrical field strength at the surface equals:

$$
\begin{aligned}
E & =\frac{1}{2} \frac{\mathrm{d} \phi}{\mathrm{d} t}=\frac{1}{2} \frac{\partial \phi}{\partial H_{\mathrm{a}}} \frac{\mathrm{d} H_{\mathrm{a}}}{\mathrm{d} t}+\frac{1}{2} \frac{\partial \phi}{\partial x_{\mathrm{t}}} \frac{\mathrm{d} x_{\mathrm{t}}}{\mathrm{d} t} \\
& =\left(\left(d-x_{\mathrm{t}}\right) \frac{\partial B_{\mathrm{en}}}{\partial H_{\mathrm{a}}}-\mu_{0} \int_{x_{\mathrm{t}}}^{d} \int_{x}^{d} \frac{\partial j_{\mathrm{c}}\left(B^{+}, x^{\prime}\right)}{\partial B^{+}} \frac{\partial B^{+}}{\partial H_{\mathrm{a}}} \mathrm{d} x^{\prime} \mathrm{d} x\right) \\
& \times \frac{\mathrm{d} H_{\mathrm{a}}}{\mathrm{d} t}
\end{aligned}
$$

For the derivation of (4), eqs. (1) and (2) have been used. When the amplitude $h_{0}$ of the ac field is taken sufficiently small, the dependence of the critical current density $i_{\mathrm{c}}$ on $B$ can be neglected within the applied field range. In this case (4) reduces to

$$
E=\left(d-x_{\mathrm{t}}\right) \frac{\partial B_{\mathrm{en}}}{\partial H_{\mathrm{a}}} \frac{\mathrm{d} H_{\mathrm{a}}}{\mathrm{d} t} .
$$

After the flux change has reached the centre plane of the sample $\left(x_{\mathrm{t}}=0\right)$ the electric field at the surface, now denoted by $E_{1}$, only depends on the total external field $H_{\mathrm{a}}$ :

$E_{1}=d \frac{\partial B_{\mathrm{en}}}{\partial H_{\mathrm{a}}} \frac{\mathrm{d} H_{\mathrm{a}}}{\mathrm{d} t}$.

The use of linearly varying (triangular or trapezoidal) fields provides a constant $\mathrm{d} H_{\mathrm{a}} / \mathrm{d} t=\alpha$; eq. (6) now immediately gives the dependence of $\partial B_{\text {en }} / \partial H_{\mathrm{a}}$ on $H_{\mathrm{a}}$. Dividing $E$ by $E_{1}$ at the same value of $H_{\mathrm{a}}$ gives:

$\frac{E}{E_{1}}=\frac{d-x_{\mathrm{t}}}{d}$

saying that at each moment $E\left(H_{\mathrm{a}}\right) / E_{1}\left(H_{\mathrm{a}}\right)$ is proportional to the position to which the flux change has entered the sample. The corresponding $B\left(x_{\mathrm{t}}, H_{\mathrm{a}}\right)$ value, which is equal to $B\left(x_{\mathrm{t}}, H_{0}-h_{0}\right)$, is given by

$B\left(x_{\mathrm{t}}, H_{\mathrm{a}}\right)=\frac{1}{2}\left[B_{\mathrm{ex}}\left(H_{0}-h_{0}\right)+B_{\mathrm{en}}\left(H_{\mathrm{a}}\right)\right]$.

Using the Taylor-expansion of $B_{\text {en }}\left(H_{\mathrm{a}}\right)$ around $H_{\mathrm{a}}$ $=H_{\mathrm{a} 0}$, eq. $(8)$ becomes in the first approximation

$B\left(x_{\mathrm{t}}, H_{\mathrm{a}}\right)=B_{\mathrm{ex}}\left(H_{0}-h_{0}\right)+\frac{1}{2}\left(H_{\mathrm{a}}-H_{\mathrm{a} 0}\right)\left(\frac{\partial B_{\mathrm{en}}}{\partial H_{\mathrm{a}}}\right)_{H_{\mathrm{a}}=H_{\mathrm{a} 0}}$,

which is linear in $H_{\mathrm{a}}=\alpha t$.

It can be concluded from (5) and (9) that when $\partial B_{\mathrm{en}} / \partial H_{\mathrm{a}}$ is independent of $H_{\mathrm{a}}$ within the applied field range, the shape of the flux profile at the moment at which the field variation started $\left(H_{\mathrm{a}}=H_{0}-h_{0}\right)$ can be seen directly from the $E$ vs. $t$ curve. When $\partial B_{\text {en }} / \partial H_{\mathrm{a}}$ is not a constant, which can be seen from those parts of the $E$ vs. $t$ curve where the flux change has reached the centre plane (6), some simple additional calculations according to (7) and (9) have to be made to obtain the actual flux distribution. When sinusoidal fields are used it is very difficult to obtain correct values for $\partial B_{\text {en }} / \partial H_{\mathrm{a}}$ from (6) because $\mathrm{d} H_{\mathrm{a}} / \mathrm{d} t$ varies continuously and even becomes zero. This may result in a less accurate determination of the flux profiles.

In the case of decreasing fields, the flux profile at the moment the external field started to decrease $\left(H_{\mathrm{a}}=H_{0}+h_{0}\right)$ can be determined in a similar way. 
Eq. (9) then becomes

$$
\begin{aligned}
& B\left(x_{\mathrm{t}}, H_{\mathrm{a}}\right)=B_{\mathrm{en}}\left(H_{0}+h_{0}\right) \\
& \quad+\frac{1}{2}\left(H_{\mathrm{a}}-H_{\mathrm{a} 0}^{\prime}\right)\left(\frac{\partial B_{\mathrm{ex}}}{\partial H_{\mathrm{a}}}\right)_{H_{\mathrm{a}}=H_{\mathrm{a} 0}^{\prime}},
\end{aligned}
$$

where $H_{\mathrm{a} 0}^{\prime}$ is given by $B_{\mathrm{ex}}\left(H_{\mathrm{a} 0}^{\prime}\right)=B_{\mathrm{en}}\left(H_{0}+h_{0}\right)$.

When a cylinder was used instead of a slab [4], eq. (5) becomes

$E=\frac{1}{2 R}\left(R^{2}-r_{\mathrm{t}}^{2}\right) \frac{\partial B_{\mathrm{en}}}{\partial H_{\mathrm{a}}} \frac{\mathrm{d} H_{\mathrm{a}}}{\mathrm{d} t}$,

where $R$ is the radius of the cylinder and $r_{\mathrm{t}}$ is the position to which the flux change has penetrated. Eq. (11) shows that the determination of $r_{t}$ is less accurate than the determination of $x_{t}$ in the case of a slab.

As was pointed out in the preceding derivation, the flux distribution inside a type II superconductor can be shown directly by plotting the voltage induced in a pick-up coil around the surface of the sample as a function of time, provided that the following conditions are satisfied:

(1) the experiment is performed on a slab in a linearly varying magnetic field; when this condition is not satisfied a considerable amount of calculation has to be done to obtain flux profiles;

(2) the behaviour of the superconductor can be described completely in terms of the CSM (see section 2) which implies that flux flow can be neglected;

(3) the critical current density $j_{\mathrm{c}}$ and also $B_{\text {eq }}\left(H_{\mathrm{a}}\right)$, $B_{\text {en }}\left(H_{\mathrm{a}}\right)$ and $B_{\text {ex }}\left(H_{\mathrm{a}}\right)$ can be understood in an effective sense, averaged over the $x-, y$ - and $z$-coordinates;

(4) the $B$-dependence of $j_{c}$, describing the flux distributions at the extreme values of the applied field $H_{0}+h_{0}$ and $H_{0}-h_{0}$, respectively, can be neglected (these two distributions need not necessarily be the same); and

(5) $\partial B_{\mathrm{en}} / \partial H_{\mathrm{a}}$ and $\partial B_{\mathrm{ex}} / \partial H_{\mathrm{a}}$ are constants within the applied field range. When this condition is not satisfied some simple additional calculations are necessary to obtain the flux distribution.

In section 5 these conditions will be discussed in comparison with our experimental results.

The use of trapezoidal fields is not only advantageous to obtain information about pinning of flux lines and surface screening, it is also very useful for superconductors in which flux flow plays a role. It was already mentioned in the introduction that various effects such as surface screening and flux flow show up at different time intervals during one cycle of the ac field whereas they mix up and mask each other in the sinusoidal case. This is indicated in fig. 4 in the case of a London-Bean superconductor [1,2]. In fig. 4 also a typical experimental curve is shown in which flux flow effects can be observed; $E$ does not vanish immediately after the external field is kept constant. After integration of $E$ to $B_{\mathrm{av}}$ this corresponds to a vertical line of length $\Delta B$ in the $B_{\mathrm{av}}$ vs. $H_{\mathrm{a}}$ curve (see fig. 2). Flux flow effects can be described in the same way as resistive effects in the normal state, using a flux flow conductivity $\sigma_{\mathrm{ff}}$. The Maxwell equations $\operatorname{read} \bar{\nabla} \times \bar{E}=-\partial \bar{B} / \partial t$ and $\bar{\nabla} \times \bar{H}=\bar{j}$, in which $\vec{j}=\bar{j}_{\mathrm{c}}+\sigma_{\mathrm{ff}} \bar{E}$ in the case of a superconductor. In a slab geometry the combination of these equations yields

$\frac{\partial^{2} B}{\partial x^{2}}=\mu_{0} \frac{\partial j_{\mathrm{c}}}{\partial x}+\mu_{0} \sigma_{\mathrm{ff}} \frac{\partial B}{\partial t}$.

In the special case of a superconductor with a constant $j_{\mathrm{c}}$ throughout the sample ( $\mathrm{d} j_{\mathrm{c}} / \mathrm{d} x=0$ ), eq. (12) reduces to the equation for the normal metal which has been studied extensively in a previous paper [16]. The boundary condition will be given by $B_{\text {en }}\left(H_{\mathrm{a}}\right)$ in increasing and $B_{\text {ex }}\left(H_{\mathrm{a}}\right)$ in decreasing field. When these

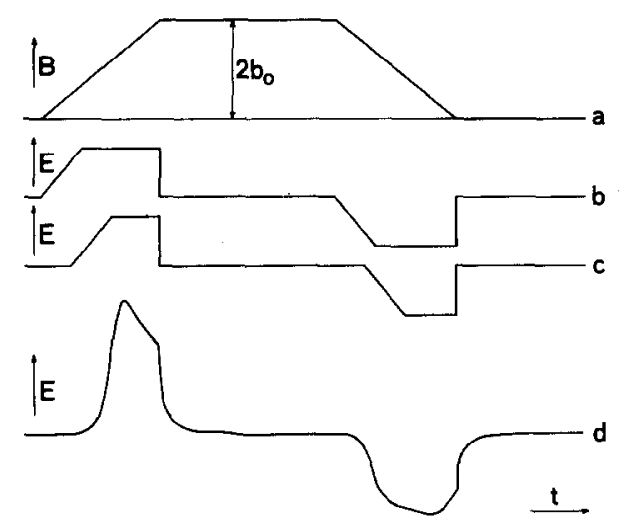

Fig. 4. (a) waveform of the applied field; (b) response curve with $j_{\mathrm{c}}=$ constant; (c) as (b) but with extra surface current contribution; and (d) typical experimental response curve (period time $25 \mathrm{~ms}, B_{0}=\mu_{0} H_{0}=0.1 \mathrm{~T}, b_{0}=35 \mathrm{mT}$ ) showing surface screening and flux flow. 
quantities vary linearly in time (e.g. $\left.\partial B_{\text {en }} / \partial t=\alpha^{\prime}\right)$ the increasing field solution of (12) in the limit for $t \rightarrow \infty$ leads to

$\Delta B=\frac{1}{3} \alpha^{\prime} \mu_{0} \sigma_{\mathrm{ff}^{\mathrm{f}}} \mathrm{d}^{2}$

which is a measure for the flux flow conductivity $\sigma_{\mathrm{ff}}$ This solution is only valid if $x_{t}=0$ and $\sigma_{\mathrm{ff}}$ is independent of the local induction $B$. Although these flux flow effects play a role during the whole cycle of the ac field the use of trapezoidal fields enables us to study them separately.

Summarizing: from the successive parts of a typical experimental response curve, as given in fig. $4 d$, the following information can be drawn:

(1) the horizontal part $(E=0)$ gives the difference between the superheating and supercooling fields;

(2) the rising part describes the flux distribution at the extreme value of the external field;

(3) the following part gives $\partial B_{\mathrm{en}} / \partial H_{\mathrm{a}}$ or $\partial B_{\mathrm{ex}} / \partial H_{\mathrm{a}}$; and

(4) the decay of $E$ to zero is a measure for the flux flow effects.

\section{Experiments}

Two types of experiments have been performed in order to investigate the behaviour of type II superconductors in trapezoidal fields. First, the response of the sample in an ac field of relatively small amplitude $\left(b_{0}<50 \mathrm{mT}\right)$, superimposed on a static field $\left(0<B_{0}\right.$ $<1.4 \mathrm{~T}$ ) was examined. The ac field was provided by a coil consisting of 300 turns of $\mathrm{Nb}$-wire, wound closely around the sample. From these experiments the flux distributions and $j_{\mathrm{c}}$-values in the whole field range between $B_{c_{1}}$ and $B_{c_{2}}$ could be obtained. Secondly, experiments were performed in trapezoidal fields with amplitudes up to $0.5 \mathrm{~T}$ without a static field. From these experiments the complete $E$ vs. $B_{\mathrm{a}}$ curve was obtained. The coil producing this field has a length of $80 \mathrm{~mm}$ and a bore of $10 \mathrm{~mm}$; it is wound of 12500 turns of $\mathrm{A61} / 05 \mathrm{CN}$ wire in 12 layers. Both types of experiments have been performed at $T=4.2 \mathrm{~K}$ [18].

The current through the ac field coils was supplied by a power amplifier. In the small amplitude experiments the trapezoidal waveform was obtained by limiting a triangular wave at the feeding voltage of the power amplifier. For the large amplitude experiments this method could only be used for very low frequencies because the self-inductance $L$ of the field coil spoiled the trapezoidal waveform. The influence of this coil-effect (described in terms of a time constant $\tau_{0}=L / R$, where $R$ is the series resistance in the circuit) on the experiments was also treated in a previous paper [16]. Since a perfectly linear time dependence of the field is necessary for the method described in section 3, a trapezium current generator was built. At the output a voltage consisting of a summation of a trapezoidal component and its derivative is generated in the correct ratio according to the resistive and the inductive component in the circuit (see fig. 5). For more details we refer to a separate publication [19].

The response signal is measured with a pick-up coil which is wound over a length of $10 \mathrm{~mm}$ around the middle section of the sample. The component of the signal due to some empty space between pick-up coil and sample, is compensated by the signal from a second pick-up coil. The empty space component is given by the derivative of the trapezoidal field and it therefore contains discontinuities which are not present in the response of the sample itself. So the absence of discontinuities in the compensated signal is a distinct criterion for correct compensation of the empty space contribution. It should be noted that this sensitive criterion is a direct consequence of the experimental method and is independent of the properties of the investigated material. This seems to be an extra advantage of our method since in the case of sinusoidal fields [20-22] flux flow response and phase shift may interfere with the compensation criterion.

Especially in the case of experiments without a static field and with an amplitude in the vicinity of the field of first penetration, the position of the compensating pick-up coil with respect to the sample turned out to be of great importance. This is due to the fact that the external field variations close to the sample differ from those far away, because of the demagnetization factor of the sample. Therefore, some experiments were done where a small compen. sating coil was inserted in the space between the pickup coil and the sample both as close to the sample surface as possible. This effect is the subject of further investigations.

After sufficient amplification, both the reference 


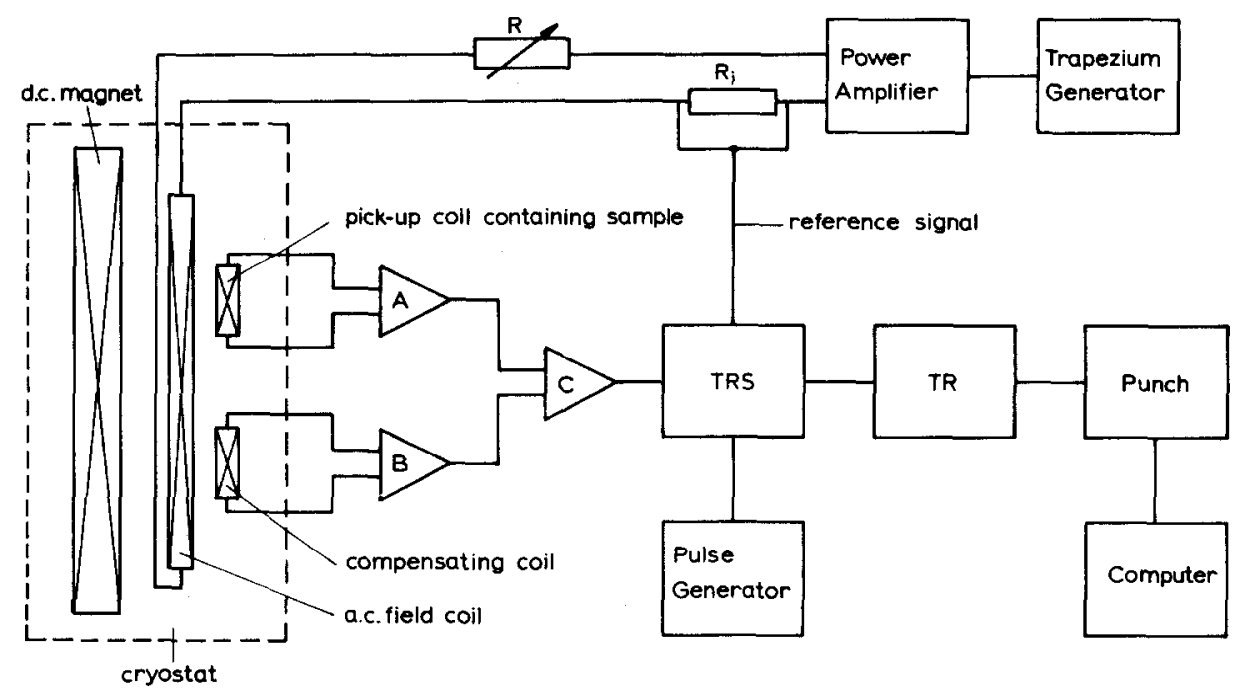

Fig. 5. Block diagram of the equipment; the letters $A$ and $B$ indicate amplifiers for measuring signal and compensating signal, respectively, while $\mathrm{C}$ is a differential amplifier.

signal and the measured signal are registrated in a memory $(\mathrm{TR}=$ transient recorder $)$ by means of a trigger-reference-signal-switch (TRS). The data then are punched on papertape and analyzed with the help of a computer $[19,23]$. A diagram of the experimental equipment is given in fig. 5 .

\section{Results and discussion}

In this section we will present our experimental results and discuss them on the basis of the theory given in the sections 2 and 3 . We will restrict ourselves mainly to the experiments performed in ac fields with a relatively small amplitude superimposed on a static field. These experiments were carried out on samples taken from commercial $\mathrm{Nb}$-plate and heat treated for 10 min at $10^{-6}$ torr just below the melting point; the surface of the material divided up into crystallites of about $2 \mathrm{~mm}^{2}$. Two samples were cut from this $\mathrm{Nb}$ plate: the larger one with dimensions $40 \times 15 \times 0.3 \mathrm{~mm}$ was used in the small amplitude experiments, the other with dimensions $30 \times 5 \times 0.3 \mathrm{~mm}$ was used in the large amplitude experiments.

In fig. $4 \mathrm{~d}$ a typical response curve for the larger sample at a static field $B_{0}=\mu_{0} H_{0}=0.1 \mathrm{~T}$ and an amplitude $b_{0}=35 \mathrm{mT}$ is shown. It can be seen from this figure that superheating and supercooling effects play a role in the sample under consideration since $E$ remains zero for some time after the external field started changing. So a distinction between $B_{\mathrm{en}}\left(H_{\mathrm{a}}\right)$ and $B_{\text {ex }}\left(H_{\mathrm{a}}\right)$ is necessary. It also can be observed from the intervals where the flux change has reached the centre plane that $\partial B_{\text {en }} / \partial H_{\mathrm{a}}$ and $\partial B_{\text {ex }} / \partial H_{\mathrm{a}}$ cannot be assumed independent of the external field. When the flux change has reached the centre plane of the sample $\left(x_{\mathrm{t}}=0\right)$, the value of $E$ (denoted by $E_{1}$ ) only depends on the total field $H_{\mathrm{a}}(4)$. Therefore values of $E_{1}$, normalized with the help of $E_{0}$, the value of $E$ in the normal case $\left(H_{\mathrm{a}} \gg H_{\mathrm{c}_{3}}\right)$, can be combined to one single $E_{1} / E_{0}$ vs. $B_{\mathrm{a}}$ curve (see fig. 6). Since $E_{0}$ is proportional to $\mathrm{d} B_{\mathrm{a}} / \mathrm{d} t$ one would expect from eq. (4) to obtain one universal $E_{1}$ vs. $B_{\mathrm{a}}$ plot for all $\mathrm{d} B_{\mathrm{a}} / \mathrm{d} t$ values. Fig. 6 shows clearly that this is not the case. This effect finds a natural explanation in the occurrence of flux flow. As was pointed out in section 3, one should account for flux flow by replacing $j_{\mathrm{c}}$ by the over-critical current density $j=j_{\mathrm{c}}+\sigma_{\mathrm{ff}} E$. This leads to a non-linear dependence of $E_{1}$ on $\mathrm{d} B_{\mathrm{a}} / \mathrm{d} t$. This explanation is supported by the decay of $E$ in the time interval where the external field is constant (see fig. 4d). In the very simple case that both $j_{\mathrm{c}}$ and $\sigma_{\mathrm{ff}}$ are independent of $B_{\mathrm{a}}$ and constant throughout the sample, eq. (13) gives an explicit relation between $\Delta B$ and $\sigma_{\mathrm{ff}}$. However, when these conditions are fulfilled, still only a single $E_{1}$ vs. $B_{\mathrm{a}}$ curve for each 


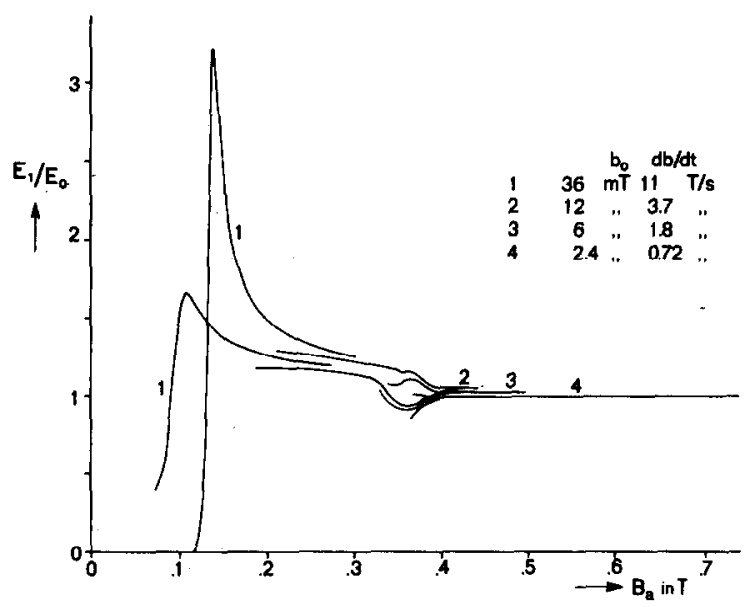

Fig. 6. The $E_{1} / E_{0}$ vs. $B_{\mathrm{a}}$ curve for increasing and decreasing field obtained by combination of small amplitude experiments; period time is $25 \mathrm{~ms}$.

$\partial B_{\mathrm{a}} / \partial t$ value should be obtained. So only in this case is a quantitative determination of $\sigma_{\mathrm{ff}}$ warranted, whereas our experimental results only allow for quantitative conclusions about the $B$-dependence of $\sigma_{\mathrm{ff}}$. A rough analysis of the shape of the decay of the $E$ vs. $t$ curve leads us to doubt the validity of the simple relation $j=j_{\mathrm{c}}+\sigma_{\mathrm{ff}} E$. Further experiments are in progress.

A typical result of the large amplitude experiment is presented in fig. 7 which shows $E$ for all values of $B_{\mathrm{a}}$ between -0.5 and $+0.5 \mathrm{~T}$ in a single measurement. The overall features of this curve are in good agreement with the results of the small amplitude experiments.

In fig. 8 some flux profiles for increasing and decreasing fields are given, as obtained with the help of eqs. (7) and (9), which implies the assumptions that the critical current density $j_{c}$ is independent of $B$ and that flux flow may be neglected. Although we have demonstrated above that these assumptions are not valid for our samples, the $B$-dependence of $j_{\mathrm{c}}$ and the presence of flux flow influence $E$ and $E_{1}$ in a similar way. Since in the determination of the flux profiles only the ratio $E / E_{1}$ is relevant, these effects will cancel in the first approximation. This consideration holds only when $E_{1}$ can be determined experimentally as a function of $B_{\mathrm{a}}$ and $\mathrm{d} B_{\mathrm{a}} / \mathrm{d} t$ which is the case in linearly varying fields. The profiles were measured with an amplitude $b_{0}=12 \mathrm{mT}$; the external field values vary between 0.242 and $0.366 \mathrm{~T}$. As was pointed

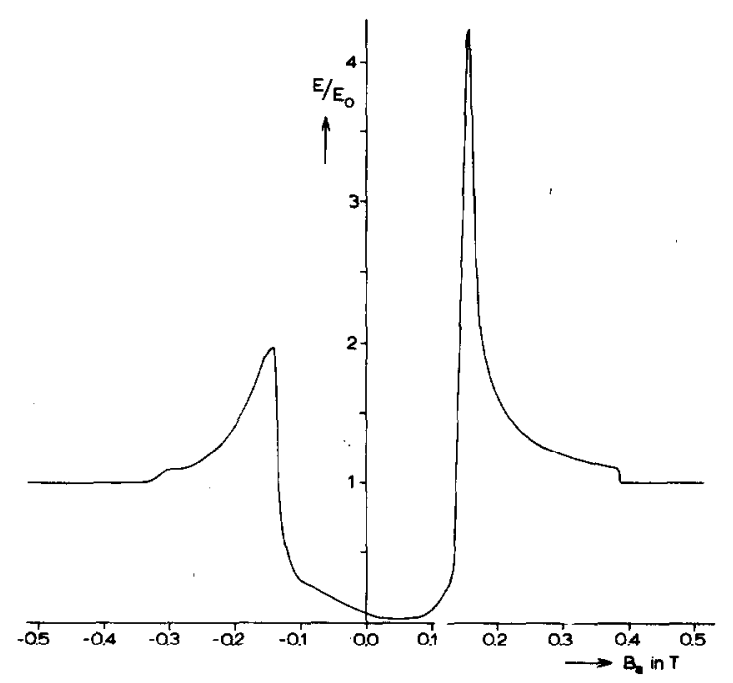

Fig. 7. The complete $E$ vs. $B_{\mathrm{a}}$ curve obtained with an ac field of large amplitude $\left(b_{0}=0.5 \mathrm{~T} ; d B_{\mathrm{a}} / \mathrm{d} t=0.85 \mathrm{~T} / \mathrm{S}\right)$. The external field clearly exceeds the second critical field $B_{\mathrm{c}_{2}}$. The values of $E$ are normalized with $E_{0}$ which corresponds tc the normal state.

out in section 3 the response in the decreasing field is determined by the flux profile at the moment when the field was at its maximum value. This profile was built up when the field was increasing.

In these flux distributions a significant difference between the pinning of flux lines in a small region close to the surface and in the rest of the bulk is observed. While the distribution of pinning centres in the bulk is almost homogeneous, there is a highly inhomogeneous surface layer of about $8 \mu \mathrm{m}$ in which the critical current density increases to about 25 times the value in the bulk. This effect, which has been reported for various types of samples $[4,17,24,25]$, is ascribed to a higher concentration of deformations and impurities near the surface. It can be seen from fig. 8 that the variations of the local induction $B$ during one cycle are small $(<15 \mathrm{mT})$, which is consistent with our assumption that for these flux profiles $j_{\mathfrak{c}}$ may be considered independent of $B$.

From the flux distributions in fig. 8 the values of the critical current density $j_{\mathrm{c}}$ for the bulk are determined; for this calculation the bulk was assumed to be homogeneous. These values, as a function of the external field $B_{\mathrm{a}}$, are given in fig. 9. The correct $j_{\mathrm{c}}(B)$ dependence cannot be given here since the $B_{\text {en }}\left(H_{\mathrm{a}}\right)$ and $B_{\mathrm{cx}}\left(H_{\mathrm{a}}\right)$ relations are not known. As expected, 

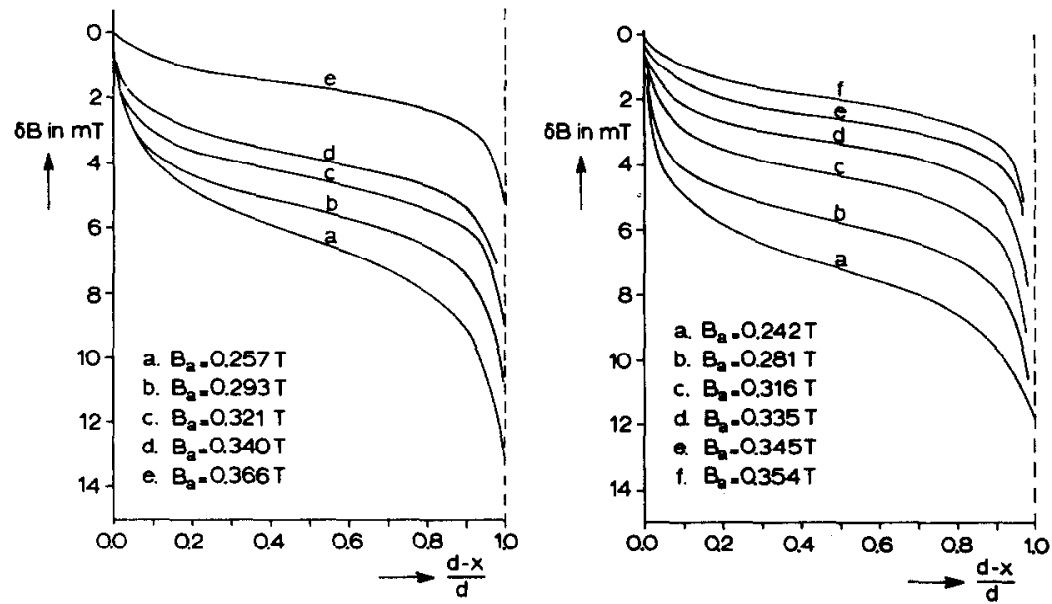

Fig. 8. Flux profiles for different values of the external field in: (a) decreasing field and (b) increasing field; the profiles were obtained with an ac field with period time $25 \mathrm{~ms}$, amplitude $b_{0}=12 \mathrm{mT}$ and $\mathrm{d} B \mathrm{a} / \mathrm{d} t=3.7 \mathrm{~T} / \mathrm{s} . \delta B$ is given by $B(x, t)$

$-B_{\mathrm{ex}}\left(H_{0}-h_{0}\right)$ in the case of a decreasing field and by $B(x, t)-B_{\mathrm{en}}\left(H_{0}+h_{0}\right)$ in the case of an increasing field.

the $j_{\mathrm{c}}$ values for increasing and decreasing field fit on the same $j_{\mathrm{c}}\left(B_{\mathrm{a}}\right)$ curve. Closer to $B_{\mathrm{c}_{1}}$ (where larger amplitudes had to be used: $b_{0}=35 \mathrm{mT}$ ) this is no longer the case as can be seen from fig. 9. This can be attributed to the fact that $j_{\mathrm{c}}$ is plotted as a function of $B_{\mathrm{a}}$ and not of $B_{\mathrm{en}}$ and $B_{\mathrm{ex}}$. The difference between the two $B_{\mathrm{a}}$ values, giving the same $j_{\mathrm{c}}$ for increasing and decreasing field, is of the same order of magnitude as the difference between $B_{\text {en }}$ and $B_{\text {ex }}$ observed in the $E$ vs. $B_{\mathrm{a}}$ loops. The obtained values are in good agreement with those obtained by Kroeger et al. [24] and Das Gupta et al. [25] for similar samples. In the high pinning region close to the surface, the value of the critical current density is much harder to determine since in this region the variations of $j_{c}$ are much larger and the flux profiles less accurate. A rough estimate, however, is obtained from fig. 8a, giving a value of about $7 \times 10^{4} \mathrm{~A} \mathrm{~cm}^{-2}$.

Near the centre plane of the sample the reduced $E$ values suggest a considerable increase of the critical current density. Such an increase of the concentration of pinning sites near the centre of the slab, however, is very unlikely. A possible explanation is that, due to an inhomogeneous distribution of pinning sites in the bulk of the sample, the flux front (that is, the place to which the flux change has entered) will not be a flat surface parallel to the $y-z$ plane. As a result the quantity $x_{t}$, used in section 3 , can only be defined in an averaged way in those regions where the flux front is connected and moving as a whole. Near the centre of the sample, where the two flux fronts meet, this averaging process is no longer defined, so $x_{t}$ looses its meaning. Therefore the calculation of the critical current density by differentiation of the reduced $E$

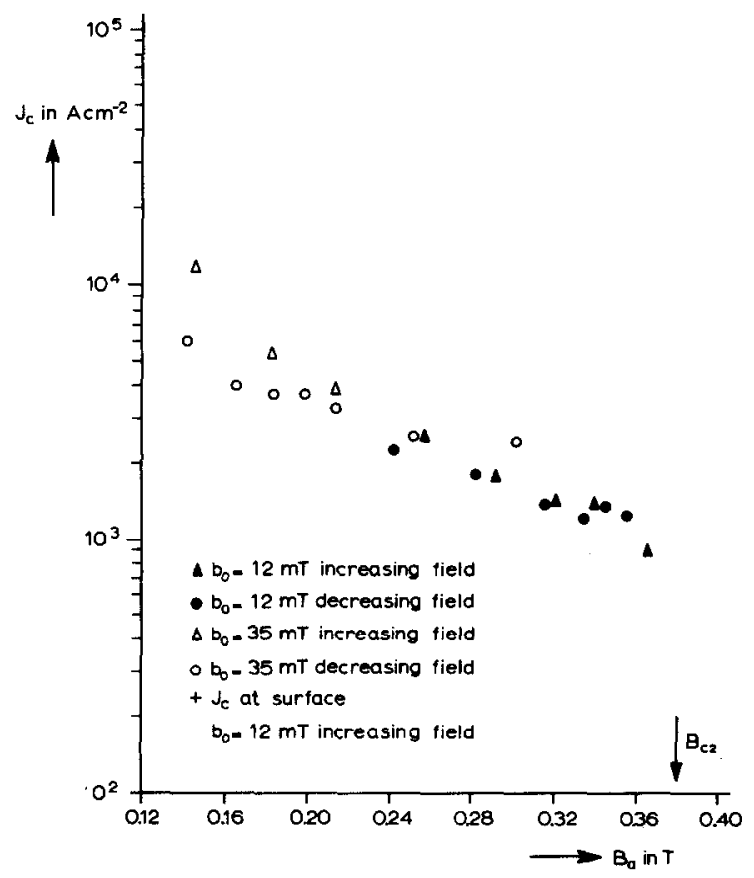

Fig. 9. Critical current density values as a function of the external field $B_{\mathrm{a}}$ as obtained from fig. 8 and from experiments with an amplitude of $35 \mathrm{mT}$. The value of $B_{c_{2}}$ is taken from fig. 6 . 
curve with respect to $x_{t}$ breaks down. Analogous considerations hold for inhomogeneous surface pinning and screening and for surface roughness, because they delay the formation of a connected flux front until some parts of the (bended) flux lines have already considerably moved in. These latter effects, however, also affect the flux profile near the surface and therefore also the meaning of $x_{t}$ in this region may be questioned. So the high values of $j_{\mathrm{c}}$ in the surface region may be spurious. The similarity of the behaviour of $E$ at the surface and in the centre region (see fig. 8) certainly suggests that the same mechanisms play a role. This would lead to the conclusion that the irregularities of the flux front extend over a distance of about $8 \mu \mathrm{m}$ and do not vary appreciably during the motion of the flux front.

\section{Conclusions}

It has been pointed out that there are several mechanisms in type II superconductors that cause hysteresis:

(a) superheating and supercooling (surface screening);

(b) pinning of flux lines at inhomogeneities at the surface (surface pinning);

(c) pinning of flux lines at deformations and impurities in the bulk of the sample (bulk pinning);

(d) viscous effects (flux flow).

The use of trapezoidal ac fields and slab geometry has proved to be very useful in obtaining information about these different hysteresis phenomena, because the various effects can be studied separately. In this paper a method, based on the CSM, is given to determine the critical current density $j_{\mathrm{c}}$ and its position dependence $j_{\mathrm{c}}(x)$. Also, the influence of other phenomena, flux flow, surface roughness and an inhomogeneous distribution of pinning sites, on this method have been discussed.

It was argued that the critical state model can also be applied in non-ideal cases with inhomogeneous pinning and surface roughness when the relevant parameters $B_{\text {en }}, B_{\text {ex }}$ and $j_{\mathrm{c}}$ are taken as effective quantities. The experimental results for the reduced voltage show that flux flow effects cannot be neglected. On the other hand it was argued that thesc flux flow effects do not interfere with the determination of the critical current density. The analysis of the flux profiles strongly suggests that the distortion of the flux line lattice and consequently a bended or disconnected flux front are the most important causes of the apparent increase of $j_{\mathrm{c}}$ at the surface and in the centre of the sample. The thickness of the irregularities of the flux front could be estimated in the order of $8 \mu \mathrm{m}$.

The small amplitude experiments presented here only give information about the difference between $B_{\text {en }}$ and $B_{\text {ex }}$ as a function of the applied field. Direct information about the $B_{\text {en }}\left(H_{\mathrm{a}}\right)$ and $B_{\text {ex }}\left(H_{\mathrm{a}}\right)$ relations can only be obtained from large amplitude experiments. The results of these experiments will be reported in due course, together with results of more detailed experiments with small amplitudes for these and other samples.

\section{Acknowledgements}

The authors express their gratitude to Prof. Dr. L. C. van der Marel for stimulating discussions and to Mr. H. A. Steffens and Mr. M. Caspari for technical assistance during the measurements.

\section{References}

[1] C. P. Bean, Phys. Rev. Lett. 8 (1962) 250.

[2] H. London, Phys. Lett. 6 (1963) 162.

[3] A. M. Campbell and J. E. Evetts, Advan. Phys. 21 (1972) 199.

[4] R. W. Rollins, H. Küpfer and W. Gey, J. Appl. Phys. 45 (1974) 5392.

[5] L. J. M. van de Klundert, E. A. Gijsbertse and L. C. van der Marel, Proc. LT 14, vol. 2 (Helsinki, 1975) p. 501.

[6] L. J. M. van de Klundert, H. T. Nijnuis, H. P. van de Braak and L. C. van der Marel, Proc. ICEC, vol. 6, (Grenoble, 1976) p. 442.

[7] C. P. Bean and J. D. Livingston, Phys. Rev. Lett. 12 (1964) 14.

[8] P. G. de Gennes, Superconductivity of Metals and Alloys (Benjamin, New York, 1966) Ch. 3, p. 76.

[9] P. G. de Gennes, Solid State Commun. 3 (1965) 127.

[10] J. Matricon and D. Saint-James, Phys. Lett. 24A (1967) 241.

[11] H. A. Ullmaier, Phys. Status Solidi 17 (1966) 631.

[12] J. R. Clem, Proc. LT 13, vol. 3 (Plenum Press, 1974) p. 102.

[13] H. J. Fink, Phys. Rev. Lett. 16 (1966) 447.

[14] P. V. Christiansen and H. Smith, Phys. Rev. 171 (1968) 445. 
[15] S. Sh. Akhmedov, V. R. Karasik and A. I. Rusinov, Zh. Eksp. Teor. Fiz. 56 (1969) 444; Sov. Phys. JETP 29 (1969) 243.

[16] L. J. M. van de Klundert, H. P. van de Braak and E. A. Gijsbertse, Physica 90B (1977) 237.

[17] A. M. Campbell, J. Phys. C2 (1969) 1492.

[18] Commercially available from IMI.

[19] L. J. M. van de Klundert and M. Caspari, to be published.

[20] J. C. Male, Gryogenics 10 (1970) 381.

[21] R. M. Easson and P. Hlawiczka, J. Appl. Phys. 18 (1967) 1237.
[22] P. Penczynski, Siemens Forsch. Entwickl. Ber. 2 (1973) 296.

[23] L. J. M. van de Klundert, C. de Rooij, M. Caspari and L. C. van der Marel, Cryogenics 15 (1975) 577.

[24] D. M. Kroeger, C. C. Koch and J. P. Charlesworth, J. Low-Temp. Phys. 19 (1975) 493.

[25] A. Das Gupta, W. Gey, J. Halbritter, H. Küpfer and J. A. Yasaitis, J. Appl. Phys. 47 (1976) 2146. 